\title{
Stellungnahme zu Schwarz E., Maier M. - Publication output in the field of Public Health and its appreciation at political level in Austria, Wien Med Wochenschr (2014) 164:123-130
}

\author{
Reaktion auf den Leserbrief des Ludwig Boltzmann Institute for Health \\ Promotion Research und Ludwig Boltzmann Institute for Health \\ Technology Assessment
}

\section{Eva Schwarz · Manfred Maier}

Online publiziert: 18. November 2014

(C) Springer-Verlag Wien 2014

Herzlichen Dank für die kritischen Anmerkungen zu unserer Publikation.

Ihr Kommentar kritisiert die für unsere Untersuchung gewählte Methodik, insbesondere die Suchstrategie, und bedauert, dass deshalb die eigene Institution nicht der Realität entsprechend mit ihrem Publikationsoutput im Bereich Public Health in unseren Ergebnissen enthalten ist.

Diese Kritik ist durchaus berechtigt: wir haben allerdings deshalb in der Diskussion mehrfach auf die methodischen Limitierungen hingewiesen und bereits im ersten Satz der Diskussion unsere Arbeit als „starting point“ und als „Pilot Studie“ bezeichnet. Insbesondere haben wir festgehalten, dass bei der gewählten Daten-

Leserbrief zur Publication output in the field of Public Health and its appreciation at political level in Austria': DOI 10.1007/s10354014-0324-7

Dr. rer. soc. oec. E. Schwarz, MPH ( $\bowtie)$

University of Applied Sciences,

Krems, Österreich

E-Mail: eva.schwarz@fh-krems.ac.at

Dr. rer. soc. oec. E. Schwarz, MPH

Centre of Public Health, Medical University of Vienna,

Wien, Österreich

E-Mail: eva.schwarz@meduniwien.ac.at

Univ. Prof. Dr. M. Maier

Department of General Practice and Family Medicine, Centre of

Public Health, Medical University of Vienna,

Wien, Österreich

E-Mail: manfred.maier@meduniwien.ac.at bank PubMed und der Suchstrategie z. Bsp. Co-Autorschaften nicht gefunden werden können und dass namentlich die beiden betroffenen „Ludwig Boltzmann Institute" mit ihren Publikationen eine andere Suchstrategie benötigen.

Da es sich bei unserer Publikation um eine Pilotstudie gehandelt hat - unseres Wissens nach die erste ihrer Art für Österreich - wäre es wünschenswert, die Studie sowohl in Richtung Erweiterung der Suchstrategie als auch der durchsuchten wissenschaftlichen Datenbanken und der Indikatoren für eine etwaige Umsetzung auszuweiten. Dafür wäre eine Kooperation zwischen den Public Health Instituten in Österreich sowie den Boltzmann Instituten und der Medizinischen Universität Wien sehr sinnvoll; wir laden daher die genannten Institutionen - wie im letzten Absatz unserer Publikation vorgeschlagen - zu einer derartigen Kooperation ein, um tatsächlich ein umfassendes und möglichst vollständiges Bild über die Publikationsleistung im Bereich Public Health in Österreich zu erhalten. Wir freuen uns über das Echo auf unsere orientierende Untersuchung und sehen einer Kooperation mit Interesse entgegen.

\section{Interessenkonflikt}

Die Autoren bestätigen, dass keine Interessenkonflikte bestehen. 